\title{
On the (di)graphs with (directed) proper connection number two ${ }^{1}$
}

\author{
Guillaume Ducoffe ${ }^{\mathrm{a}, \mathrm{b}}$ Ruxandra Marinescu-Ghemeci ${ }^{\mathrm{c}, \mathrm{d}}$ \\ Alexandru Popa ${ }^{\mathrm{b}, \mathrm{c}}$ \\ a Université Côte d'Azur, Inria, CNRS, I3S, France \\ b National Institute for Research and Development in Informatics, Romania \\ ${ }^{\mathrm{c}}$ University of Bucharest, Faculty of Mathematics and Computer Science \\ d The Research Institute of the University of Bucharest ICUB, Romania
}

\begin{abstract}
A coloring of a graph $G$ is properly connected if every two vertices of $G$ are the ends of a properly colored path. We study the complexity of computing the proper connection number (minimum number of colors in a properly connected coloring) for edge and vertex colorings, in undirected and directed graphs, respectively. First we disprove some conjectures of Magnant et al. (2016) on characterizing the strong digraphs with proper arc connection number at most two. Then, we prove that deciding whether a given digraph has proper arc connection number at most two is NP-complete. We initiate the study of proper vertex connectivity in digraphs and we prove similar results as for the arc version. Finally, we present polynomialtime recognition algorithms for bounded-treewidth graphs and bipartite graphs with proper edge connection number at most two.
\end{abstract}

Keywords: proper connection; digraphs; bipartite; even dicycles; NP-complete.

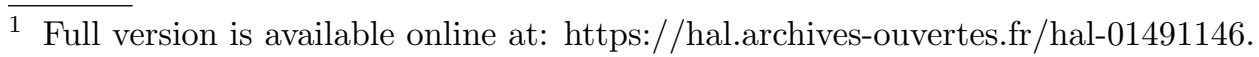




\section{Introduction}

We study a relaxed variant of proper colorings, introduced by Borozan et al. [2], where we only impose for every two vertices $u, v$ to have a properly colored (di)path from $u$ to $v$ - the (di)graph itself may not be properly colored. The latter concept is sometimes called proper connectivity. Properly colored paths have applications in many fields like genetics [7] or communication networks. As an example, it is desirable in wireless networks to have all the parties connected and to avoid interference by ensuring that the incoming and the outgoing signal from a tower should be on different frequencies. Suppose that we assign a vertex to each signal tower, an edge between two vertices if the corresponding signal towers are directly connected by a signal and a color to each edge corresponding to the frequency used for the communication. Then, the number of frequencies needed to assign the connections between towers so that there is always a path avoiding interference between each pair of towers is precisely the proper connection number of the corresponding graph.

RELATED WORK. The proper connection number in undirected graphs (for edge colorings) was first defined in [2] by Borozan et al., where they relate the proper connection number with the graph connectivity. Since then the problem was intensively studied from the combinatorial point of view $[1,2,9,15,13]$. In particular, bounds on the proper connection number of random graphs and bipartite graphs have been proved, respectively, in [9] and $[2,11,10]$. Relationships between proper connection number and domination number can be found in [13]. Many generalizations of proper connectivity have been proposed $[2,11,1,14]$. For instance, in this paper, we also study some notions of vertex proper connection, i.e., vertex-coloring versions of the proper connection number (see [6]). More recently, Magnant et al. studied the proper arc connection number for strong digraphs [15]. They proved that this number is always at most three and they asked to characterize the digraphs with proper connection number at most two. In particular, they conjectured that all such digraphs must contain an even dicycle. For more details, see the following survey on the proper edge connection number: [12]. Nevertheless, no complexity results have been proved for proper connectivity, until our work. Our goal is to fill in this gap in the literature.

Proper connectivity is related to the rainbow connectivity, defined in [5]. Since computing the rainbow connection number is NP-hard and not FPT for any fixed $k \geq 2$ [3] it is natural to study the complexity of computing the proper edge connectivity - which can be seen a relaxed variant of rainbow connectivity. 


\section{Our results}

We study the complexity of computing the proper edge (resp., vertex, resp., arc) connection number of a given (di)graph. This gives four parameters to study, that are respectively denoted by $p c_{e}(G)$ and $p c_{v}(G)$ for undirected graphs $G ; \overrightarrow{p c_{e}}(D)$ and $\overrightarrow{p c_{v}}(D)$ for directed graphs $D$. In this section we give an overview of our results (summarized in Table 1). Most of the proofs are omitted due to lack of space.

Observe that deciding whether $p c_{e}(G) \leq k$ (resp., $p c_{v}(G) \leq k, \overrightarrow{p c_{e}}(D) \leq$ $\left.k, \overrightarrow{p c_{v}}(D) \leq k\right)$ is in NP since, as a certificate for yes-instances, it suffices to give a properly connected coloring with at most $k$ colors and, for every ordered pair $u, v$ of vertices, a properly colored (di)path from $u$ to $v$. First we study the complexity of computing $\overrightarrow{p c_{e}}(D)$ for a given digraph $D$. In [15] Magnant et al. prove that $\overrightarrow{p c_{e}}(D) \leq 3$ for every strong digraph $D$.

Theorem 2.1 Deciding whether $\overrightarrow{p c_{e}}(D) \leq 2$ for a digraph $D$ is NP-complete.

Our proof of Theorem 2.1 uses a reduction from Positive NAE 3-SAT, the variant of 3-SAT in which all the variables are unnegated, and the extra requirement is that in every clause, the variables should not all have the same valuation. Second, we disprove a conjecture of Magnant et al. from [15], claiming that if $D$ is a strong digraph with no even dicycle, then $\overrightarrow{p c_{e}}(D)=3$.

Theorem 2.2 There exists an infinite family of digraphs with no even dicycles that also have properly connected 2-colorings.

The construction of the infinite family that is stated in Theorem 2.2 is based on a properly connected 2-coloring of $D_{7}$, that is the only strongly biconnected digraph with no even dicycle [16]. Then we turn our attention to the computational complexity of computing $\overrightarrow{p c_{v}}(D)$ for a given digraph $D$. To the best of our knowledge, the proper vertex connection number of directed graphs has not been studied before.

Theorem 2.3 For every strong digraph $D, \overrightarrow{p c_{v}}(D) \leq 3$. However, deciding whether $\overrightarrow{p c_{v}}(D) \leq 2$ is NP-complete.

The reduction of Theorem 2.3 is from 3-SAT instead of Positive NAE 3SAT. Furthermore although the two hardness proofs of Theorems 2.1 and 2.3 are similar, notice that none of the two results is implied by the other. Finally, we study the proper connection numbers of undirected graphs.

Theorem 2.4 Deciding whether $p c_{e}(G) \leq 2$ is in $P$ when $G$ is bipartite or has a bounded treewidth. 


\begin{tabular}{|c|c|c|}
\hline & Directed & Undirected \\
\hline \multirow{2}{*}{ Edges } & NP-hard to decide if $\overrightarrow{p c_{e}}(D) \leq 2$ & $p c_{e}(G) \leq 2$ in P for $G$ bipartite \\
\cline { 2 - 3 } & $\overrightarrow{p c}_{e}\left(D_{7}\right)=2$ & $p c_{e}(G) \leq 2$ in P for $G$ bounded tw \\
\hline \multirow{2}{*}{ Vertices } & $\overrightarrow{p c_{v}}(D) \leq 3$ if $D$ is strongly connected & Trivial \\
\cline { 2 - 2 } & NP-hard to decide if $\overrightarrow{p c_{v}}(D) \leq 2$ & \\
\hline
\end{tabular}

Table 1

For bounded treewidth graphs, the result is obtained by applying Courcelle's Theorem (the $\mathrm{MSO}_{2}$ formula is a variant of the one for Hamiltonian РАтн). The algorithm for bipartite graphs is presented in the next section. The complexity of deciding whether $p c_{e}(G) \leq 2$ for general graphs is left as an interesting open question.

\section{Proper edge connection number: a polynomial case}

Several sufficient conditions have been given for bipartite graphs to have proper connection number equal to two $[2,4,8,10,11]$. We base on one such a known condition (i.e., Lemma 3.2) in order to provide a complete characterization of the bipartite graphs with proper connection number two. In what follows, the bridge-block tree of $G$ is the tree with nodes the bridge-blocks of $G$ (2-edge-connected components) and, for every bridge of $G$, an edge between the two bridge-blocks that contain its ends. It is linear-time computable [17].

Theorem 3.1 Let $G=(V, E)$ be a connected bipartite graph. We have $p c_{e}(G) \leq 2$ if and only if the bridge-block tree of $G$ is a path. Furthermore, if $p c_{e}(G) \leq 2$, then such a coloring can be computed in linear-time.

The sufficient part and the necessary part of the proof of Theorem 3.1 are based on Lemmas 3.2 and 3.3, respectively. In what follows, we say that an edge-coloring of $G$ has the strong property if, for every two vertices $u$ and $v$, there are at least two $u v$-paths (not necessarily disjoint) with the two edges being incident to $u$ (resp., to $v$ ) in the two paths being of different colors.

Lemma 3.2 ([11]) If $G$ is a connected bipartite bridgeless graph, then $p c_{e}(G) \leq$ 2. Furthermore, such a coloring can be produced with the strong property.

Hence, by Lemma 3.2, it remains to study bipartite graphs with bridges. For that we need the following lower-bound:

Lemma 3.3 Let $G=(V, E)$ be a connected graph, $B$ be a bridge-block of $G$ that is bipartite. If $B$ is incident to at least three bridges then $p c_{e}(G) \geq 3$. 
The proof of Lemma 3.3 is based on a parity argument (see Figure 1). More precisely, we prove that, in any properly connected coloring of $G$ with two colors: all the bridges incident to the same side of the bipartition of $B$ must have different colors; while two bridges incident to different sides of the bipartition of $B$ must have the same color. If there are

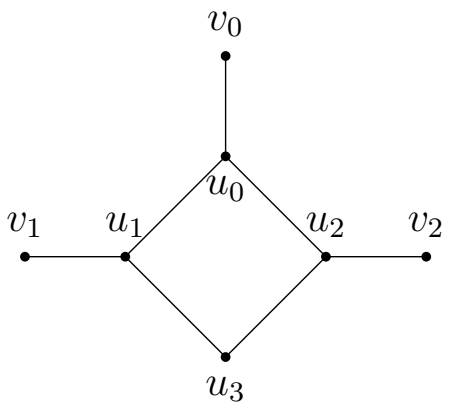

Figure 1: $p c_{e}(G)=3$. at least three bridges incident to $B$, then it gives a contradiction.

Proof sketch of Theorem 3.1. On the one direction, suppose that the bridgeblock tree of $G$ is not a path. Edges in this tree are in one-to-one correspondance with bridges in $G$. So, there is a bridge-block of $G$ that is incident to at least three bridges. Since such a component must be bipartite (because $G$ is), it follows from Lemma 3.3 that $p c_{e}(G) \geq 3$.

On the other direction, suppose that the bridge-block tree of $G$ is a path. This gives us a linear ordering $B_{0}, B_{1}, \ldots, B_{l}$ over the bridge-blocks. We compute, independently for every $0 \leq i \leq l$, a properly connected 2-coloring of $G\left[B_{i}\right]$ with the strong property, that exists by Lemma 3.2. Then, if $l>0$, we assign an arbitrary color to the unique bridge between $B_{0}$ and $B_{1}$; for every $2 \leq i \leq l$, we assign a color to the unique bridge between $B_{i-1}$ and $B_{i}$ that depends on the color previously assigned to the unique bridge between $B_{i-2}$ and $B_{i-1}$ (we use the parity argument of Lemma 3.3 in order to decide the color). Since, for every $0 \leq i \leq l$, the 2-coloring of $G\left[B_{i}\right]$ has the strong property, it can be verified that the 2-coloring of $G$ so obtained is properly connected.

\section{References}

[1] Andrews, E., C. Lumduanhom, E. Laforge and P. Zhang, On proper-path colorings in graphs, J. Comb. Math. and Comb. Comp. 97 (2016), pp. 189207.

[2] Borozan, V., S. Fujita, A. Gerek, C. Magnant, Y. Manoussakis, L. Montero and Z. Tuza, Proper connection of graphs, Discrete Mathematics 312 (2012), pp. 2550-2560.

[3] Chakraborty, S., E. Fischer, A. Matsliah and R. Yuster, Hardness and 
algorithms for rainbow connection, J. of Combinatorial Optimization 21 (2011), pp. 330-347.

[4] Chang, H., Z. Huang and X. Li, Degree sum conditions for graphs to have proper connection number 2, Technical Report arXiv:1611.09500, arXiv (2016).

[5] Chartrand, G., G. L. Johns, K. A. McKeon and P. Zhang, Rainbow connection in graphs, Mathematica Bohemica 133 (2008), pp. 85-98.

[6] Chizmar, E., C. Magnant and P. Salehi Nowbandegani, Note on vertex and total proper connection numbers, AKCE International J. of Graphs and Combinatorics 13 (2016), pp. 103-106.

[7] Dorninger, D., Hamiltonian circuits determining the order of chromosomes, Discrete Applied Mathematics 50 (1994), pp. 159-168.

[8] Gerek, A., S. Fujita and C. Magnant, Proper connection with many colors, J. of Combinatorics 3 (2012), pp. 683-693.

[9] Gu, R., X. Li and Z. Qin, Proper connection number of random graphs, Theoretical Computer Science 609 (2016), pp. 336-343.

[10] Huang, F., X. Li, Z. Qin and C. Magnant, Minimum degree condition for proper connection number 2, Theoretical Computer Science (2016).

[11] Huang, F., X. Li and S. Wang, Proper connection number and 2-proper connection number of a graph, arXiv preprint arXiv:1507.01426 (2015).

[12] Li, X. and C. Magnant, Properly colored notions of connectivity-a dynamic survey, Theory and Applications of Graphs (2015), p. 2.

[13] Li, X., M. Wei and J. Yue, Proper connection number and connected dominating sets, Theoretical Computer Science 607 (2015), pp. 480-487.

[14] Lumduanhom, C., E. Laforge and P. Zhang, Characterizations of graphs having large proper connection numbers, Discussiones Mathematicae Graph Theory $\mathbf{3 6}$ (2016), pp. 439-453.

[15] Magnant, C., P. R. Morley, S. Porter, P. S. Nowbandegani and H. Wang, Directed proper connection of graphs, МАТЕМАТИЧКИ ВЕСНИК 68 (2016), pp. $58-65$.

[16] McCuaig, W., Even dicycles, J. of Graph Theory 35 (2015), pp. 46-68.

[17] Tarjan, R. E., A note on finding the bridges of a graph, Information Processing Letters 2 (1974), pp. 160-161. 